\title{
Candida albicans promotes tooth decay by inducing oral microbial dysbiosis
}

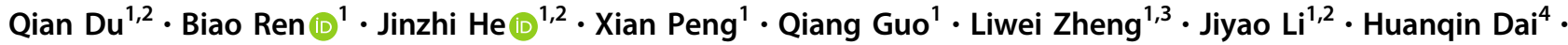 \\ Vivian Chen ${ }^{1} \cdot$ Lixin Zhang $\mathbb{1}^{5,6} \cdot$ Xuedong Zhou $\mathbb{1}^{1,2} \cdot$ Xin Xu $\mathbb{1}^{1,2}$
}

Received: 30 April 2020 / Revised: 14 October 2020 / Accepted: 22 October 2020 / Published online: 4 November 2020

(c) The Author(s), under exclusive licence to International Society for Microbial Ecology 2020

\begin{abstract}
Candida albicans has been detected in root carious lesions. The current study aimed to explore the action of this fungal species on the microbial ecology and the pathogenesis of root caries. Here, by analyzing C. albicans in supragingival dental plaque collected from root carious lesions and sound root surfaces of root-caries subjects as well as caries-free individuals, we observed significantly increased colonization of $C$. albicans in root carious lesions. Further in vitro and animal studies showed that $C$. albicans colonization increased the cariogenicity of oral biofilm by altering its microbial ecology, leading to a polymicrobial biofilm with enhanced acidogenicity, and consequently exacerbated tooth demineralization and carious lesion severity. More importantly, we demonstrated that the cariogenicity-promoting activity of $C$. albicans was dependent on PHR2. Deletion of PHR2 restored microbial equilibrium and led to a less cariogenic biofilm as demonstrated by in vitro artificial caries model or in vivo root-caries rat model. Our data indicate the critical role of $C$. albicans infection in the occurrence of root caries. PHR2 is the major factor that determines the ecological impact and caries-promoting activity of $C$. albicans in a mixed microbial consortium.
\end{abstract}

These authors contributed equally: Qian Du, Biao Ren

Supplementary information The online version of this article (https:// doi.org/10.1038/s41396-020-00823-8) contains supplementary material, which is available to authorized users.

$\triangle$ Lixin Zhang

zhanglixin@im.ac.cn

$\triangle$ Xuedong Zhou zhouxd@scu.edu.cn

$\triangle \mathrm{Xin} X \mathrm{Xu}$

xin.xu@scu.edu.cn

1 State Key Laboratory of Oral Diseases, National Clinical Research Center for Oral Diseases, West China Hospital of Stomatology, Sichuan University, 610041 Chengdu, China

2 Department of Cariology and Endodontics, West China Hospital of Stomatology, Sichuan University, 610041 Chengdu, China

3 Department of Pediatric Dentistry, West China Hospital of Stomatology, Sichuan University, 610041 Chengdu, China

4 State Key Laboratory of Mycology, Institute of Microbiology, Chinese Academy of Sciences, 100101 Beijing, China

5 Chinese Academy of Science Key Laboratory of Pathogenic Microbiology and Immunology, Institute of Microbiology, 100101 Beijing, China

6 State Key Laboratory of Bioreactor Engineering, East China University of Science and Technology, 200237 Shanghai, China

\section{Introduction}

Dental caries, also known as tooth decay, is one of the most common oral diseases that cause increasing socioeconomic burden to the humans. According to the reports from Global Burden of Diseases, Injuries, and Risk Factors Study 2016, dental caries ranked number one in prevalence and number two in incidence of the ten causes with highest prevalence/ incidence [1]. Root caries is a subtype of dental caries that mainly affects elder populations. The prevalence of root caries in the elderly reaches $25-100 \%$ [2]. Root caries occurs in areas where the root surface is exposed to the oral environment. As a clinical subtype of dental caries, root caries is a polymicrobial infectious disease associated with the enrichment of acidogenic/aciduric species (e.g., Streptococcus mutans) and depletion of less aciduric commensal residents (e.g., Streptococcus sanguinis) in the microbial biofilm [3]. The "competitive exclusion" of $S$. mutans and $S$. sanguinis has been observed in both in vivo and in vitro studies [4-6]. In addition, a positive correlation between the risk of dental caries and $S$. mutans/S. sanguinis has been observed [7, 8], further suggesting the critical role of microbial disequilibrium in the course of dental caries. Consistently, a recent study has reported microbial dysbiosis in root-caries lesions [9], further 
supporting the role of microbial ecology in the development of root caries. Another study on the microbial metatranscriptome in caries revealed that $S$. sanguinis was metabolically active in 3 types of caries lesions (i.e., noncavitated, open dentin, and hidden caries), while $S$. mutans was active only in noncavitated and open dentin caries [10], suggesting the role of oxygen availability in the interspecies interactions between these two bacteria.

Candida albicans is a commensal fungal species commonly colonizing the human mucosal surfaces. The oral carriage of $C$. albicans in healthy individuals is $18.5-40.9 \%$ based on cultivation [11-13]. Carriage rates are usually higher in individuals with compromised immunity, such as human immunodeficiency virus-positive individuals, diabetes patients, and infants and elder populations [11, 12, 14-16]. Hyphal formation and the ability to respond to extreme environmental $\mathrm{pH}$ levels are essential for $C$. albicans pathogenicity [17-19]. In addition, $C$. albicans can robustly interact with other oral microorganisms and impose significant impact on the virulence of polymicrobial biofilms. The microbial interaction and cross-kingdom feeding between $C$. albicans and oral bacteria such as Streptococcus, Actinomyces, and Fusobacterium species have been suggested being closely associated with the pathogenesis of oral infectious diseases for years [20-24]. Cross-kingdom interactions between C. albicans and Streptococcus oralis enhance biofilm virulence on mucosal surfaces [25-27]. In addition, a positive correlation between $C$. albicans prevalence/carriage and the severity of early childhood caries (ECC) has been noted [28-30], and $C$. albicans is associated with an increased abundance of plaque S. mutans in severe ECC $[31,32]$. Findings by Falsetta et al. demonstrated that interactions between $C$. albicans and $S$. mutans not only increased bacterial-fungal carriage in the plaque but also enhanced the biofilm virulence by increasing the severity of carious lesions in a rodent model [33]. More importantly, $C$. albicans has been isolated from root caries lesions [34-36], and observed in carious dentin/dentine tubules [37]. Genes involved in metabolic activity, sugar transport, stress tolerance, invasion and $\mathrm{pH}$ regulation are upregulated in $C$. albicans colonized in root carious lesion as compared to $C$. albicans in sound root surface [38], further underscoring the potential role of $C$. albicans in the development of root caries.

Although the association of $C$. albicans with root caries has been suggested, the exact action of this fungal species on the microbial ecology and the pathogenesis of root caries is yet to be investigated. In addition, key factors that determine the successful colonization of $C$. albicans and the subsequent cross-kingdom interactions with other microorganisms in the polymicrobial biofilms are not clear. Here, we hypothesize that $C$. albicans may affect the microbial ecology and increase the cariogenicity of oral biofilms, and thus promote the development of root caries. Specifically, we found increased colonization of $C$. albicans in root carious lesions. C. albicans colonization imposed significant ecological impact on oral microbiota, leading to a polymicrobial biofilm with enhanced cariogenicity. PHR2 was identified as the major factor that determined the ecological impact and caries-promoting activity of $C$. albicans, which provided a promising target for the control of root caries.

\section{Materials and methods}

\section{Clinical sample analyses}

To investigate the association between $C$. albicans and root caries occurrence/microbial composition, we enrolled seventy volunteers aged 55-85 years old (35 root-caries subjects and 35 caries-free subjects) in this study. The study protocol was reviewed and approved by the Institution Review Board of West China Hospital of Stomatology, Sichuan University (WCHSIRB-D-2016-100). Written informed consent was obtained from all participants in the study. None of the volunteers had taken or received daily medicine, antibiotic treatment, hormone treatment or oral treatment within 3 months prior to the study. Dental plaque was collected from root carious lesions of root-caries molars and sound root surfaces of molars lacking carious lesions of root-caries patients, as well as the sound root surfaces of all molars of caries-free subjects.

Sample size was estimated using the following assumption: type I error equals 5\%; type II error equals $10 \%$; percentage of people infected oral $C$. albicans equals $30 \%$; percentage of root-caries subjects infected oral $C$. albicans equals $72 \%$. Based on these assumptions and using PASS 13.0 statistical software, the minimum required sample size in the root-caries subjects or caries-free controls was 30, so we included 35 subjects per group in our study.

Pooled saliva was additionally collected from ten cariesfree healthy subjects without $C$. albicans infection (confirmed by PCR) for the establishment of saliva-derived biofilm. Each subject was instructed to spit $5 \mathrm{ml}$ of saliva directly into a sterile collection tube. Saliva samples were then pooled together and centrifuged at $2600 \times g$ for $10 \mathrm{~min}$ to spin down large debris and eukaryotic cells. The supernatant was referred to as pooled saliva and used for the establishment of saliva-derived biofilm throughout this study. Part of the collected saliva was further filtered at $0.22 \mu \mathrm{m}$ filters, and the resulting cell-free saliva was used to coat enamel blocks and glass coverslips prior to growing the in vitro biofilms. See Supplementary file for details.

Genomic DNA of dental plaque samples were isolated using QIAamp DNA micro kit (Qiagen, Valencia, CA, USA) with additional lysozyme $(3 \mathrm{mg} / \mathrm{ml}, 1.5 \mathrm{~h})$ treatment. The DNA quality was evaluated with a NanoDrop 2000 
spectrophotometer (Thermo Fisher Scientific, Waltham, MA, USA). The detection rate of $C$. albicans was determined by PCR. S. mutans, S. sanguinis, C. albicans counts were quantified using qPCR. The quantification of specific microbe was further normalized by the total DNA amount of each clinical sample. See Supplementary file for details (Supplementary Tables S1 and 2).

\section{In vitro biofilm model}

S. mutans UA159, S. sanguinis ATCC10556, and C. albicans SC5314 were commercially obtained from the American Type Culture Collection, and the mutants of $C$. albicans used in this study were listed in Supplementary Table S3. The in vitro saliva-derived biofilm and threespecies biofilm was incubated in SHI medium at $37^{\circ} \mathrm{C}$ under anaerobic conditions for $24 \mathrm{~h}$ [39, 40]. For salivaderived biofilm, $30 \mu \mathrm{l}$ of pooled saliva and $2 \times 10^{2} \mathrm{cell} / \mathrm{ml}$ C. albicans or its mutants were inoculated into $1500 \mu \mathrm{SHI}$ medium. For three-species biofilm, inoculum for the experiment was adjusted to $2 \times 10^{6} \mathrm{CFU} / \mathrm{ml}$ of $S$. mutans, $2 \times 10^{7} \mathrm{CFU} / \mathrm{ml}$ of $S$. sanguinis and $2 \times 10^{4} \mathrm{cell} / \mathrm{ml}$ of $C$. albicans or its mutants (bacterial inoculum with similar ratio to which was detected in clinical samples).

After $24 \mathrm{~h}$ incubation, the biofilms were dispersed by sonication, and a dilution series of cell suspensions were prepared and spread on selective culture plates. The total colony-forming unit (CFU) of viable $S$. mutans and $S$. sanguinis was counted on Mitis Salivarius Agar plate (MSA, Hopebio, Qingdao, China) after $48 \mathrm{~h}$ anaerobic incubation at $37^{\circ} \mathrm{C}$, and the $\mathrm{CFU}$ of viable $S$. mutans was counted on the MSA plate supplemented with $0.2 \mathrm{U} / \mathrm{ml}$ bacitracin and $20 \%$ sucrose. C. albicans or its mutants were selectively cultured with CHROMagar Candida plate (Difco Laboratories) aerobically at $35^{\circ} \mathrm{C}$ for $48 \mathrm{~h}$ before CFU counting. Genomic DNA of biofilms was also isolated using QIAamp DNA micro kit (Qiagen) with additional lysozyme $(3 \mathrm{mg} / \mathrm{ml}, 1.5 \mathrm{~h}$ ) treatment. Total RNA of yeast was isolated using Yeast RNAiso Kit (TaKaRa, Shiga, Japan). Total bacterial RNA from saliva-derived biofilm was isolated using TriZol Reagent (Invitrogen, Carlsbad, CA, USA) [41]. The DNA and RNA qualities were evaluated with a NanoDrop 2000 spectrophotometer (Thermo Fisher Scientific). The amount of each microbe and the expression levels of PHR1, PHR2, CRZ1, CRZ2, RIM101, RIM8, UME6, TPK1, TPK2, CPH1, CPH2, ldh, arcA, and ureC were determined by qPCR. See Supplementary file for details (Supplementary Tables S2, 4, and 5).

\section{S rRNA sequencing and analyses}

16s rRNA sequencing was performed to investigate the ecological impact of $C$. albicans or its PHRI/PHR2 deletion mutants on saliva-derived biofilm. PCR amplification of the nearly full-length bacterial $16 \mathrm{~S}$ rRNA genes was performed using the forward primer (5'-AGAGTTTGATCMTGGCTC AG- $3^{\prime}$ ) and reversed primer ( $5^{\prime}$-ACCTTGTTACGACTT- $3^{\prime}$ ). A unique 12-mer tag for each DNA sample was added to the $5^{\prime}$-end of both primers to pool multiple samples together. The amplicons were sequenced by the PacBio Sequel platform (Shanghai Personal Biotechnology Co., Ltd, Shanghai, China). An average of 5000 reads per sample was generated from the amplicon library. Sequence data analyses were mainly performed using QIIME (v1.8.0) and R packages ( $\mathrm{v}$ 3.2.0), including quality control of raw data, taxonomic annotation according to NCBI database. The data were further analyzed as follows: (1) Shannon diversity index was calculated among groups. (2) The weighted UniFrac principal coordinates analysis $(\mathrm{PCOA})$ and dissimilarity tests including Adonis and ANOSIM were used to examine the community differences between groups. (3) Co-occurrence analysis was performed by calculating Spearman's rank correlations between predominant taxa. Correlations with $\rho>0.6$ and $P<$ 0.01 were constructed into an association network and imported into Cytoscape software (http://www.cytoscape.org/) for visualization [42]. (4) The relative abundance difference of bacterial taxa between groups was compared by KruskalWallis test at both the genus and species levels. (5) Microbial functions were predicted by Phylogenetic investigation of communities by reconstruction of unobserved states based on the 16S rRNA gene sequence data [43].

\section{In vitro three-species biofilm imaging and structural analyses}

To visualize the composition and structure of the biofilm, $C$. albicans or its mutants was stained with Calcofluor White (Sigma-Aldrich, St. Louis, MO, USA) [44], and fluorescent in situ hybridization (FISH) was performed as described previously to label $S$. mutans and S. sanguinis with speciesspecific probes (Supplementary Table S6) [45]. The microbe cells and extracellular polysaccharides (EPS) were labeled with SYTO 9 (Molecular Probes, Invitrogen) and Alexa Fluor 647-labeled dextran conjugate (Molecular Probes, Life Technologies, Grand Island, NY, USA) respectively [46]. Biofilm images were captured and analyzed with a laser scanning confocal microscope equipped with Fluoview SV1000 imaging software (Olympus FV1000, Tokyo, Japan). The population of microbes and EPS were analyzed based on integral optical density (IOD) with Image pro plus 6.0 (Media Cybernetics). See Supplementary file for details.

\section{In vitro artificial caries model}

To evaluate the demineralization-promoting capability of $C$. albicans and its mutants on saliva-derived biofilm, the 
in vitro artificial caries model was established by seeding saliva-derived biofilm with/without $C$. albicans on human enamel blocks in SHI medium for 5 days and refreshed $1 \mathrm{ml}$ medium every $24 \mathrm{~h}$. The $\mathrm{pH}$ values of the spent media were measured with a pH meter (Thermo Fisher Scientific). The $\mathrm{NH}_{3}$ level in the medium was determined with an ammonia assay kit (Jiancheng, Bioengineering institute, Nanjing, China) at the end of a 5-day period. After 5-day incubation, the enamel blocks were prepared for transversal microradiography (TMR) analysis [47]. See Supplementary file for details.

\section{In vivo root-caries rat model}

A root-caries rat model was used to further investigate the role of $C$. albicans and its mutants in the pathogenesis of root caries. The animal protocol was reviewed and approved by the animal research committee of West China School of Stomatology, Sichuan University (WCHSIRB-D-2017113). The rat model was established as previously described with modifications (Supplementary Fig. S1) [48]. Male specific-pathogen-free Sprague-Dawley rats (aged 17 days and weighing $60 \pm 5 \mathrm{~g}$ ) were purchased from Dashuo Inc. (Chengdu, China), and were provided with cariogenic diet. Rats ( $n=5$ per group) were either infected with $C$. albicans or $S$. mutans/S. sanguinis, S. mutans/S. sanguinis/C. albicans, S. mutans/S. sanguinis/phr $2 \Delta / \Delta, S$. mutans/S. sanguinis/phrl $\Delta / \Delta$ with simple randomization. Rats without infection were also included as control. The rat mouths were infected by respective microbe with the same ratio as they were in the three-species biofilm as stated above. Gingivectomies were performed for all rats on day 10 after initial infection. Animals were sacrificed 6 weeks after initial infection. The dental plaque was collected for qPCR quantification, and the jaws were collected for root-caries scoring using Doff's system [49]. The determination of the root-caries score was blinded by codification of the jaws. See Supplementary file for details.

We estimated the sample size considering the variation and means of the samples calculated from the preliminary experiment. The type I error equals 5\%, type II error equals $10 \%$. Based on these assumptions and using PASS 13.0 statistical software, the minimum required sample size in each group was 5 .

\section{Statistical analyses}

Each in vitro experiment was repeated three times. Statistical analysis of data, other than those obtained from microbiome sequencing, was performed with SPSS (v 16.0). Detection rates of $C$. albicans and quantitative analyses of microbial composition in the fluorescence-labeled three-species biofilm were analyzed with chi-squared test. Data obtained from clinical samples, including quantification of $C$. albicans, $S$. mutans, $S$. sanguinis and $S$. mutans/S. sanguinis ratio were analyzed with Kruskal-Wallis test followed by post hoc Dunn's test due to asymmetrical distributions, and results are presented as median with interquartile range. Linear correlation and regression were also calculated between the level of C. albicans and $S$. mutans or $S$. mutans/S. sanguinis ratio in clinical samples. After test for homogeneity of variance, other data obtained from two independent groups were analyzed with $t$-test, and multiple group comparisons were performed by one-way analysis of variance test followed by Tukey's test to compare means of each group, and Dunnett $t$-test to compare the means of all other groups with the control group, and results are presented as mean \pm standard deviation. Data were considered significantly different if the two-tailed $P$ value was $<0.05$. Sample sizes for each experiment are detailed in the figure legends.

\section{Results}

\section{Root carious lesions harbor increased amount of $C$. albicans and S. mutans}

The microbial composition of supragingival dental plaque collected from root carious lesions was compared with that of sound root surfaces of root-caries subjects as well as cariesfree controls. We found that $C$. albicans colonized more frequently at root carious lesions rather than at sound root surfaces of the same patients with root caries and the caries-free individuals $(71.4 \%, 31.4 \%$, and $28.6 \%, P<0.05$; Fig. 1a). Further quantification by qPCR showed that $C$. albicans was significantly enriched at root carious lesions [15.015 (27.386) copies/ng] compared to the sound root surfaces of root-caries patients [2.644 (8.738) copies/ng, $P<0.05]$ and the caries-free controls [0.135 (0.150) copies/ng, $P<0.0001$; Fig. 1b]. In parallel, the root-caries lesions also showed an increased carriage of $S$. mutans $\left[2.697 \times 10^{3}\left(6.284 \times 10^{3}\right)\right.$ copies $\left./ n g\right]$ as compared to the sound root surfaces of root-caries patients $\left[5.419 \times 10^{1}\left(2.412 \times 10^{2}\right)\right.$ copies/ng, $\left.P<0.0001\right]$ and the caries-free controls $\left[1.060 \times 10^{1}\left(4.296 \times 10^{1}\right)\right.$ copies/ng, $P<$ 0.0001 ; Fig. 1c]. A positive correlation was observed between the carriage of $C$. albicans and $S$. mutans $(r=0.561, P<0.01$; Fig. 1d). Conversely, a decreased amount of commensal $S$. sanguinis was observed in the root-caries lesions as compared to the caries-free controls (Fig. 1e). We further calculated the ratio of $S$. mutans/S. sanguinis, which was significantly higher in root carious lesions than that of the caries-free group $(P<$ $0.0001)$, as well as that obtained from the sound root surfaces of root-caries patients $(P<0.001$; Fig. 1f). More importantly, the $S$. mutans/S. sanguinis ratio showed a better positive correlation with the carriage of $C$. albicans detected at the same sites $(r=0.759, P<0.01$; Fig. $1 \mathrm{~g})$. 

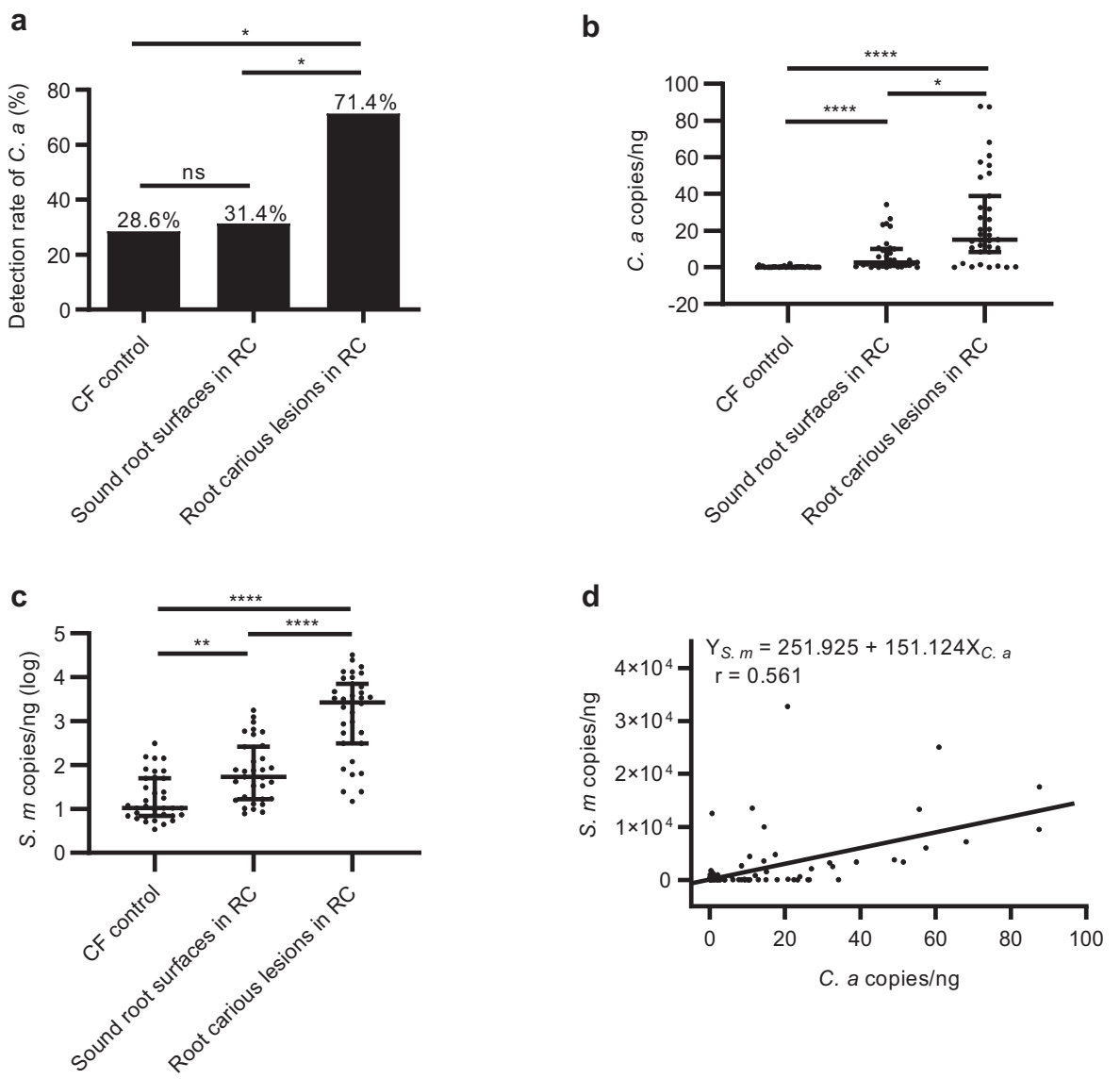
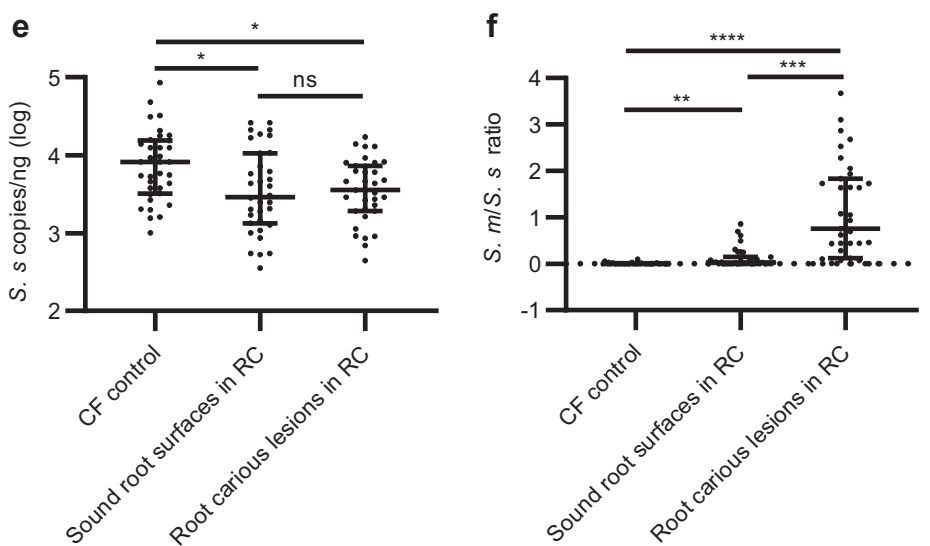

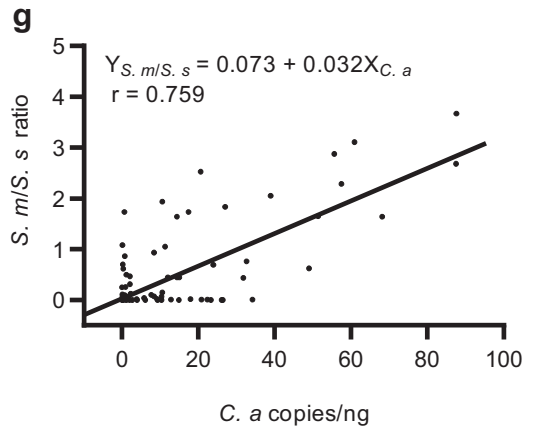

Fig. 1 Root carious lesions harbor increased amount of $C$. albicans and $S$. mutans. a The detection rate of $C$. albicans by PCR in the root carious lesions, as well as on the sound root surfaces of root-caries patients and caries-free individuals. b, $\mathbf{c}$ qPCR quantification of the amount of $C$. albicans and $S$. mutans in the collected clinical samples. d Linear correlation and regression between the amount of $C$. albicans and $S$. mutans in the supragingival plaque of all recruited subjects. e qPCR quantification of the amount of $S$. sanguinis in the collected

\section{C. albicans alters the microbial ecology of oral biofilm}

To investigate the ecological impact of $C$. albicans on the polymicrobial oral biofilm, we inoculated $C$. albicans in a clinical samples. f The calculated $S$. mutans/S. sanguinis ratio of the corresponding samples. $\mathrm{g}$ Linear correlation and regression between the amount of $C$. albicans and $S$. mutans/S. sanguinis ratio in the supragingival plaque of all recruited subjects. The results are presented as median with interquartile range (a-c, e, f: $n=35$ per group; d, $\mathbf{g}$ : $n=105 ; * P<0.05 ; * * P<0.01 ; * * * P<0.001 ; * * * * P<0.0001$; ns not significant). RC root-caries group; $\mathrm{CF}$ caries-free group; $S$. s $S$. sanguinis; S. m S. mutans; C. a C. albicans.

saliva-derived biofilm. Data obtained from full-length $16 \mathrm{~S}$ rRNA sequencing showed that $C$. albicans colonization imposed significant ecological impact on the oral biofilms. The $\alpha$-diversity of oral biofilm was unaltered in the presence of C. albicans (Fig. 2a). However, the weighted PCoA 
a

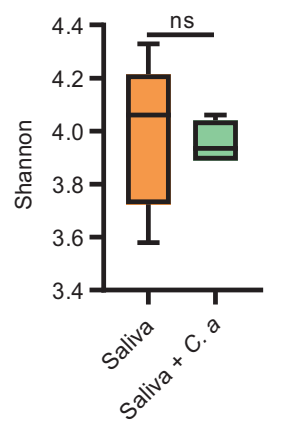

b

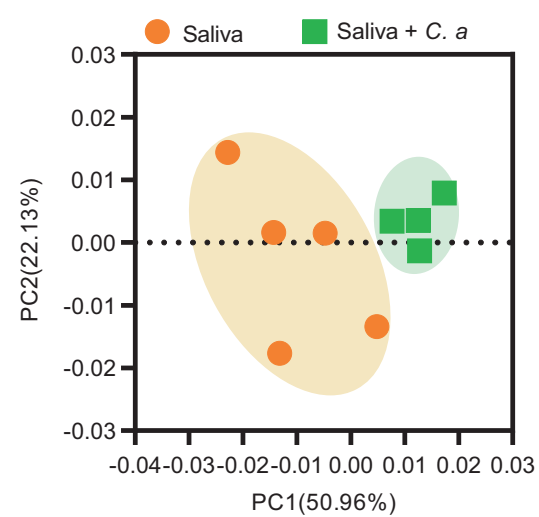

C

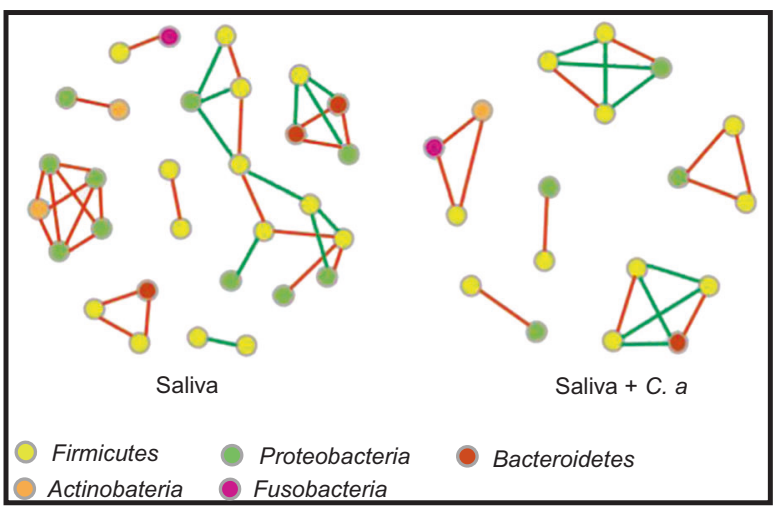

d

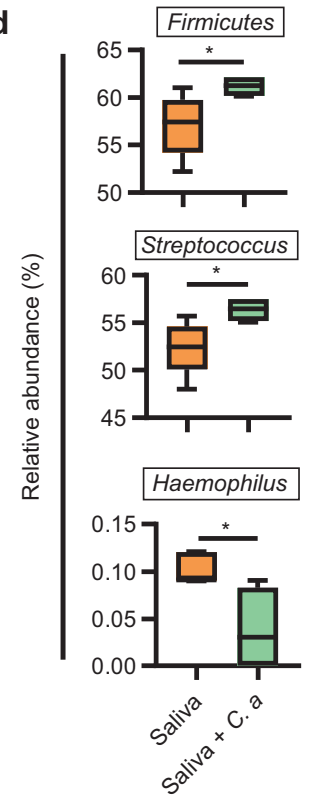

f
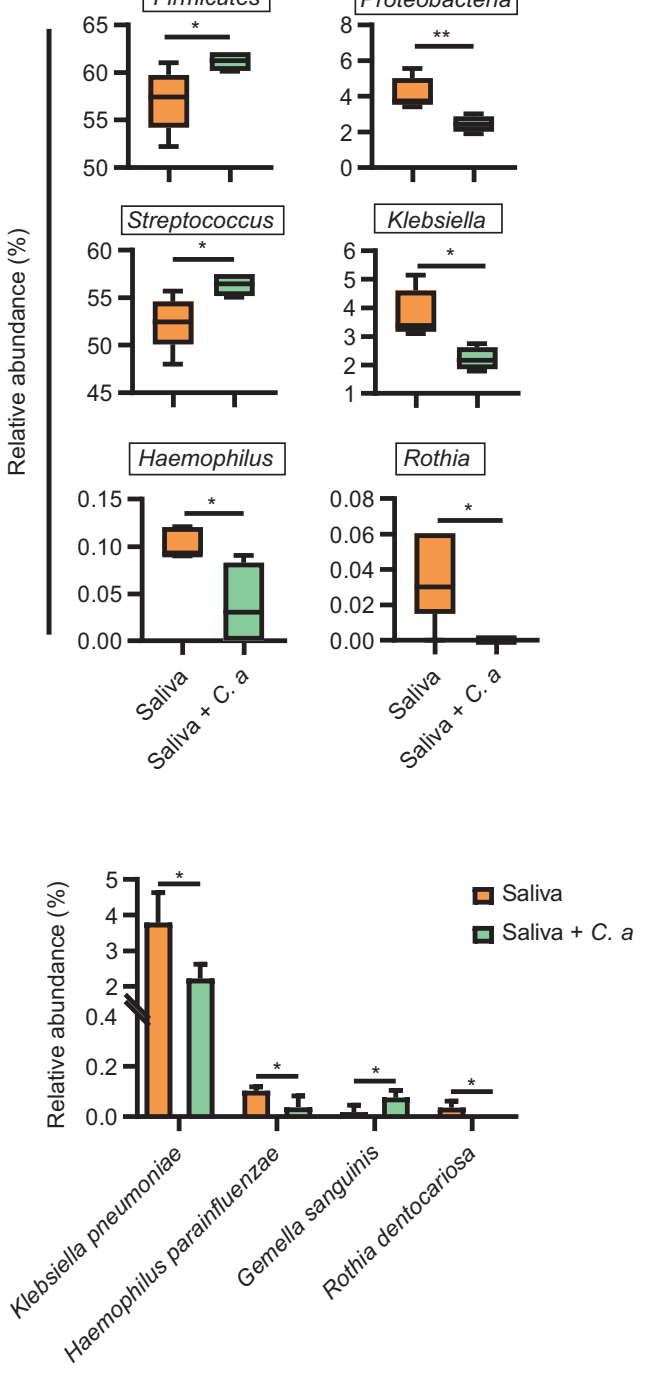

Proteobacteria

g
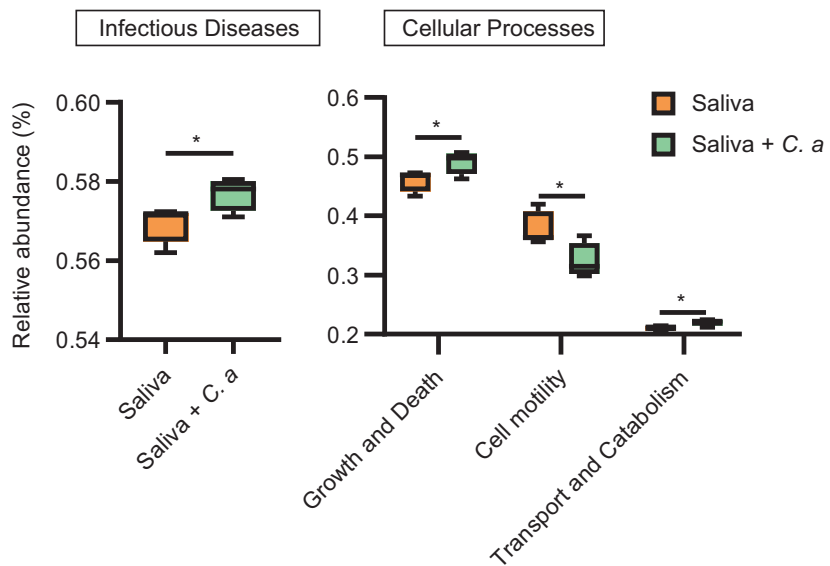

Table 1). We also constructed co-occurrence ecological networks at the species level to better delineate how salivaderived biofilm assemble and whether $C$. albicans colonization made significant impact on the oral microbial

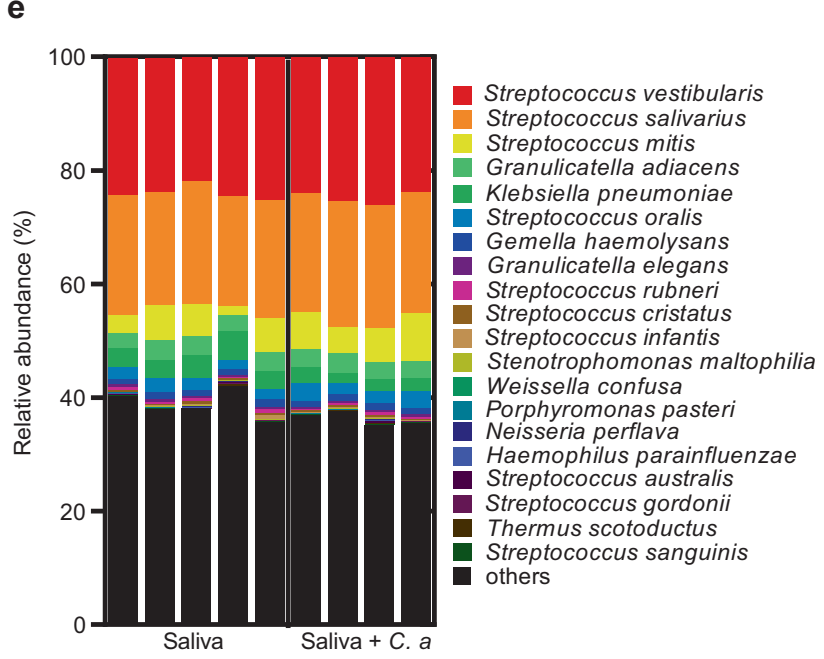

based on Bray-Curtis distance (Fig. 2b) and dissimilarity tests including Adonis and ANOSIM showed distinct microbial clusters with/without $C$. albicans (ANOSIM: $R=$ $0.5562, \quad P=0.019 ; \quad$ Adonis: $R^{2}=0.34198, \quad P=0.012$; 
Fig. 2 C. albicans alters the microbial ecology of oral biofilms. a The Shannon indices and $\mathbf{b}$ the PCoA analysis on the polymicrobial oral biofilm in the absence/presence of $C$. albicans. c Network inferences of microbial relationships in biofilms. Each node represents an OTU, and each edge represents a significant pairwise association. Red lines indicate positive relationships while green lines represent negative relationships. d The relative abundance of the significantly different phyla and genera in the saliva-derived biofilm with/without $C$. albicans. e Relative abundance of the 20 most predominant oral bacteria at species level and $\mathbf{f}$ relative abundance of the significantly different species in the oral biofilm with/without $C$. albicans. g Prediction of Kyoto Encyclopedia of Genes and Genomes (KEGG) pathways of oral biofilm in the absence/presence of $C$. albicans. The results are presented as mean \pm standard deviation $(n=4$ or 5 per group; $* P<0.05 ; * * P<0.01$; ns not significant). $S$. s $S$. sanguinis; $S$. $m$ S. mutans; C. a C. albicans.

community network topology. Specifically, co-culture with C. albicans resulted in a salivary microbiome network of fewer nodes and links, and the negative relationship between species was slightly reduced (Fig. 2c).

At phylum level, $C$. albicans significantly increased the growth of Firmicutes and decreased the growth of Proteobacteria (Fig. 2d). At genus level, C. albicans significantly improved the growth of Streptococcus and decreased the growth of Klebsiella, Haemophilus, and Rothia (Fig. 2d). Consistently, at species level, C. albicans significantly decreased the abundance of Klebsiella pneumoniae, Haemophilus parainfluenza, and Rothia dentocariosa and increased the abundance of Gemella sanguinis (Fig. 2e, f). Furthermore, the saliva-derived biofilm when co-culture with $C$. albicans, showed increased representation of predicted Kyoto Encyclopedia of Genes and Genomes pathways involved in human infectious disease and cellular processes including cell growth and death as well as transport and catabolism, and cell motility-associated pathway was decreased (Fig. 2g). All these data indicate that $C$. albicans could impose significant ecological impact on the oral microbiome.

\section{C. albicans robustly interacts with oral streptococci in the polymicrobial biofilm}

As the sequencing data showed an increased abundance of Streptococcus in the presence of $C$. albicans, we further quantified the carriage of $C$. albicans and $S$. mutans $/ S$. sanguinis in the oral biofilms. Co-culture of $C$. albicans with oral biofilms synergistically promoted the growth of both $C$. albicans and $S$. mutans, while the growth of $S$. sanguinis was unchanged as demonstrated in either salivaderived biofilms (Fig. 3a, b) or three-species biofilms by FISH, qPCR quantification (Fig. $3 \mathrm{c}$ and Supplementary Fig. S2a, b) as well as viable CFU counting (Supplementary Fig. S2c, d). Accordingly, the ratio of $S$. mutans/S. sanguinis was increased in the presence of C. albicans (Fig. 3d,
Table 1 Dissimilarity tests (Adonis and ANOSIM) for the biofilm microbiota.

\begin{tabular}{|c|c|c|c|c|}
\hline & \multicolumn{2}{|l|}{ ANOSIM } & \multicolumn{2}{|l|}{ Adonis } \\
\hline & $R$ & $P$ & $R^{2}$ & $P$ \\
\hline Over all & 0.2963 & 0.008 & 0.31515 & 0.003 \\
\hline Saliva VS Saliva $+C$. albicans & 0.5562 & 0.019 & 0.34198 & 0.012 \\
\hline Saliva VS Saliva $+p h r 2 \Delta / \Delta$ & -0.136 & 0.914 & 0.05396 & 0.968 \\
\hline Saliva VS Saliva $+p h r l \Delta / \Delta$ & 0.352 & 0.036 & 0.25467 & 0.016 \\
\hline
\end{tabular}

Supplementary Fig. S2e, f). In addition, the biofilm generated more EPS in the presence of $C$. albicans as shown by laser scanning confocal microscopy (Fig. 3e). EFGl- and CPH1-deficient mutants of $C$. albicans were constructed to further investigate if the candidal-streptococcal interactions in the polymicrobial biofilm were hyphae-dependent. Deletion of either $E F G 1$ or $C P H 1$ did not affect the carriage of S. mutans, however, C. albicans and S. sanguinis in the polymicrobial biofilm were unchanged or slightly decreased as compared to the WT strain (Fig. 3f, g and Supplementary Fig. S2g-j). In parallel, the $S$. mutans/ S. sanguinis ratio was unchanged by the deletion of EFGl and CPH1 compared with the biofilm co-cultured with the $C$. albicans WT strain (Fig. 3h and Supplementary Fig. S2k, 1).

\section{C. albicans enhances the cariogenicity of oral biofilm and promotes the development of root caries}

We further investigated if the altered microbial ecology in the presence of $C$. albicans could lead to increased cariogenicity of oral biofilm. C. albicans alone was not acidogenic in our culture conditions. However, it significantly promoted the acidogenicity of the saliva-derived biofilm as reflected by a lower supernatant $\mathrm{pH}$ along with decreased ammonia production relative to the $C$. albicansfree biofilm (Fig. 4a, b). Consistently, C. albicans significantly upregulated acid production-associated gene $l d h$, and downregulated ammonia production-associated genes $\operatorname{arcA}$ and $u r e C$ of the oral biofilm (Fig. 4c). In addition, data obtained from TMR showed that inoculation of $C$. albicans significantly increased the demineralized lesion depth and mineral loss of tooth hard tissue caused by the saliva-derived biofilm in the in vitro artificial caries model (Fig. 4d-f).

We employed a root-caries rat model to validate the role of $C$. albicans in the pathogenesis of root caries in vivo. Rats co-infected with $C$. albicans, S. mutans, and S. sanguinis showed increased microbial carriage in the dental plaque and an exacerbated development of root caries. Specifically, infection with $C$. albicans alone caused nearly no root caries, while co-infection with $C$. albicans/S. mutans/S. sanguinis caused significantly more severe root caries compared to the rats infected with $S$. mutans/S. sanguinis (Fig. 4g, h). A 
a

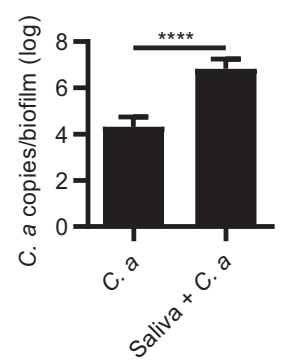

\section{C}
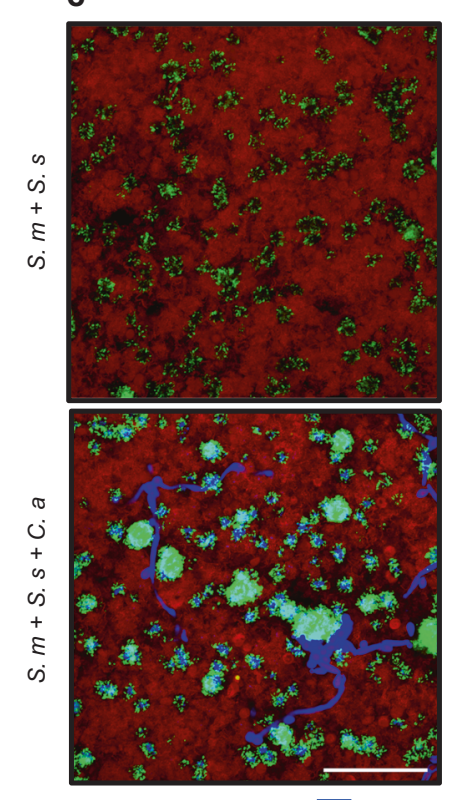

S. $m$

S.s

C. $a$

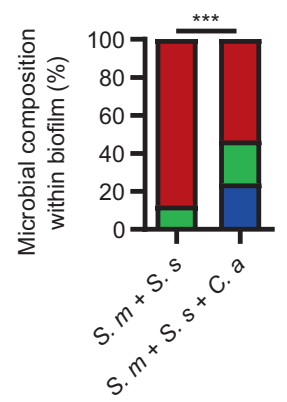

b

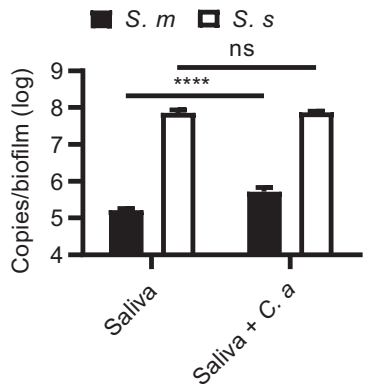

e
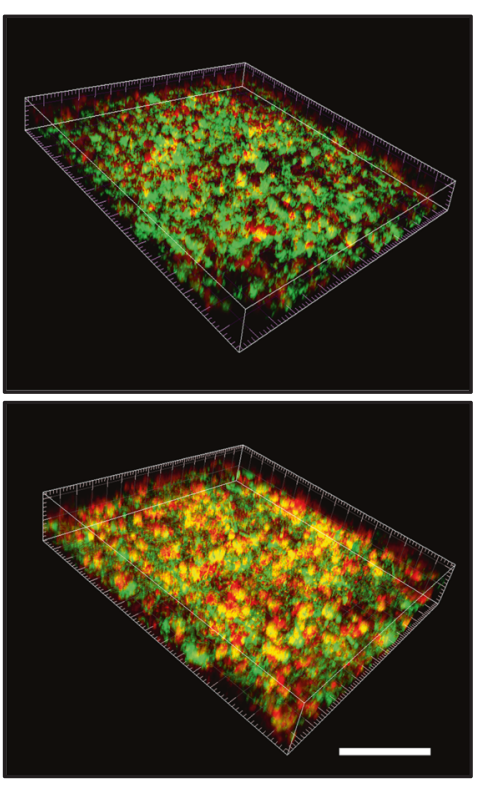

microbe EPS

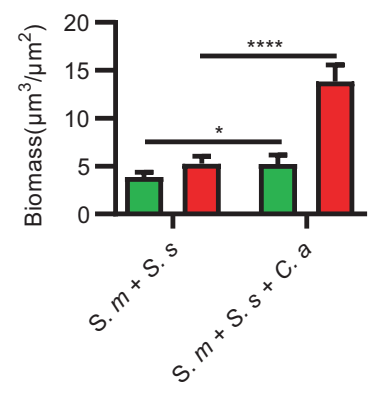

d

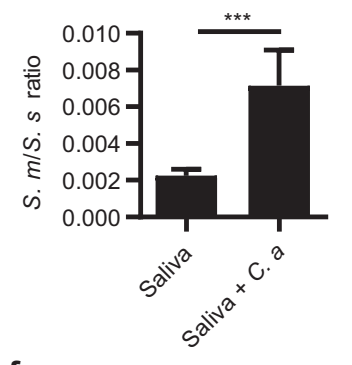

f
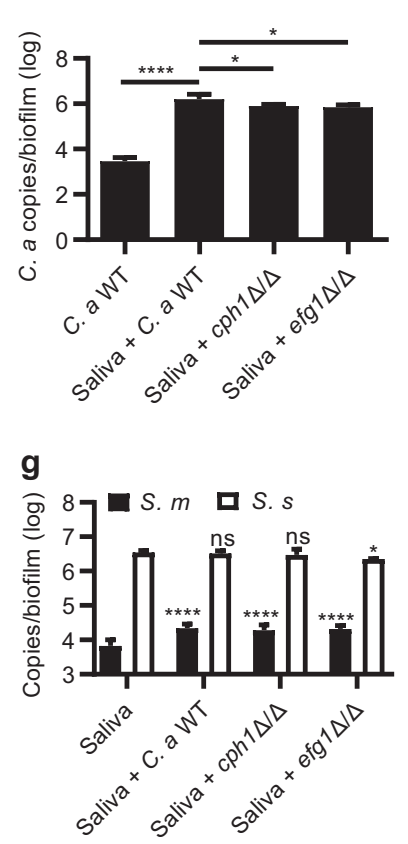

h

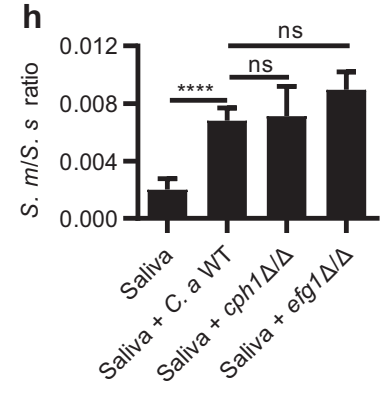

Fig. 3 C. albicans robustly interacts with oral streptococci in the polymicrobial biofilm. a, b qPCR quantification of the amount of $C$. albicans, $S$. mutans and $S$. sanguinis in the saliva-derived biofilm. c Representative images of fluorescence-labelled S. mutans (green), $S$. sanguinis (red), and C. albicans (blue) within three-species biofilm and quantitative analyses of microbial composition. Scale bar, $60 \mu \mathrm{m}$. d The $S$. mutans/S. sanguinis ratio in the saliva-derived biofilm quantified by qPCR. e Representative confocal images of double fluorescence-labelled three-species biofilm. Bacteria and C. albicans

significant increased carriage of C. albicans (16.4-fold) was observed in rats co-infected with $C$. albicans $/ S$. mutans $/ S$. sanguinis as compared to the group only infected with $C$. albicans (Fig. 4i). In parallel, the C. albicans/S. mutans/S. were labelled green, EPS was labelled red, and quantitative analyses of microbe/EPS within biofilm. Scale bar, $100 \mu \mathrm{m}$. f-h qPCR quantification of $C$. albicans or its $E F G 1 / C P H 1$ deletion mutants, S. mutans and $S$. sanguinis in the saliva-derived biofilm, and the corresponding $S$. mutants/S. sanguinis ratio. The control group of $(\mathbf{g})$ was saliva-derived biofilm. The results are presented as mean \pm standard deviation $(n=5$ per group; $* P<0.05 ; * * * P<0.001 ; * * * * P<0.0001$; ns not significant). S. s S. sanguinis; S. $m$ S. mutans; C. a C. albicans.

sanguinis co-infection group showed increased colonization of $S$. mutans as compared to the $S$. mutans/S. sanguinis infected group (Fig. 4j). In addition, the $S$. mutans/S. sanguinis ratio was also increased in the presence of $C$. albicans 


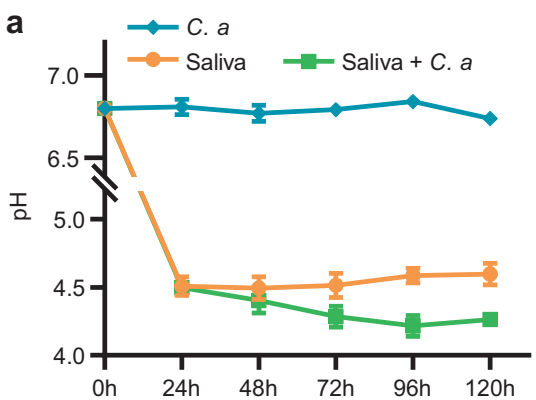

d

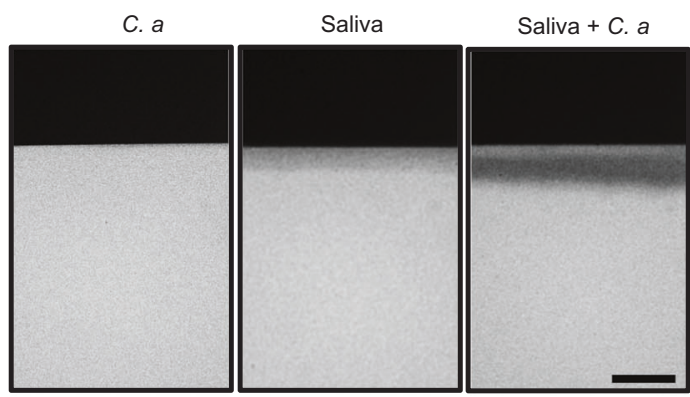

e

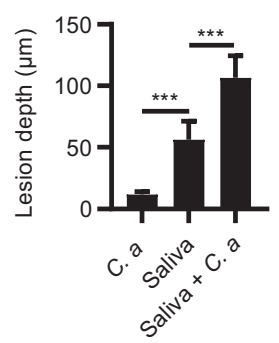

b

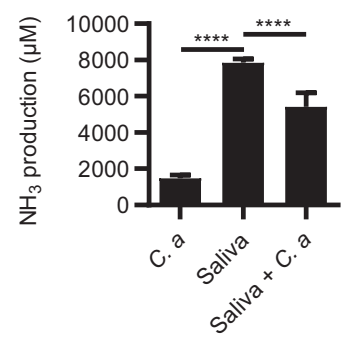

g

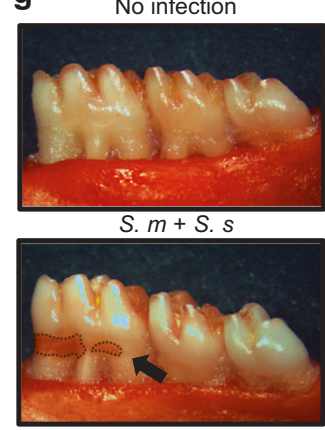

i

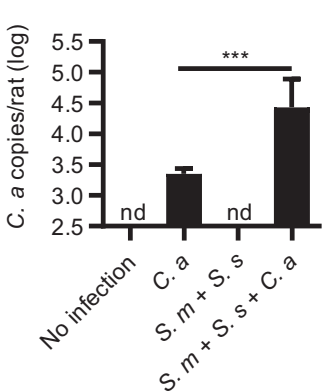

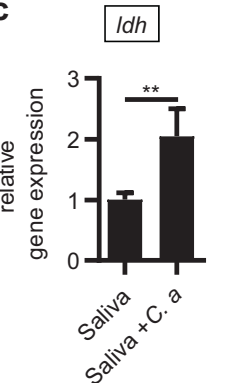

$\operatorname{arcA}$

ureC

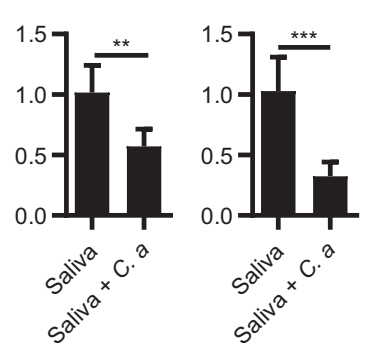

h
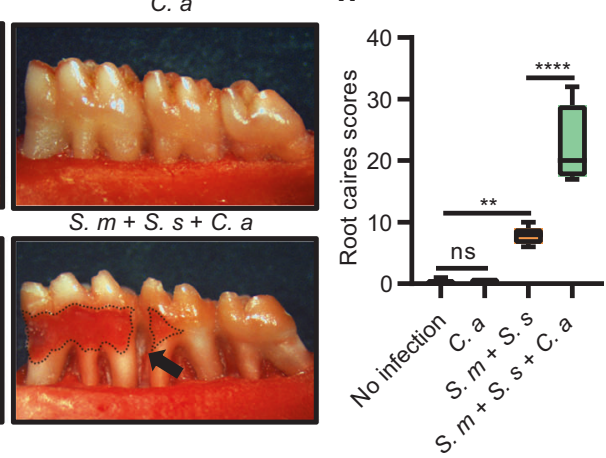

j

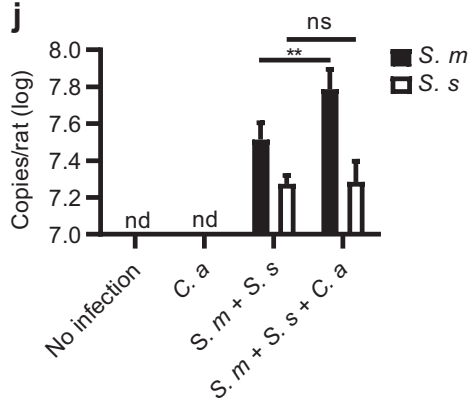

Fig. 4 C. albicans enhances the cariogenicity of oral biofilm and promotes the development of root caries. a Dynamic $\mathrm{pH}$ values of the spent media of saliva-derived biofilm in the absence/presence of $C$. albicans. b $\mathrm{NH}_{3}$ production by the saliva-derived biofilm on the 5th day after inoculation. c Relative expression of $l d h$, $\operatorname{arcA}$, and $u r e C$ of the $24 \mathrm{~h}$ saliva-derived biofilm. d Representative transversal microradiography (TMR) images of human enamel blocks demineralized by saliva-derived biofilm in the absence/presence of $C$. albicans. Scale bar, $100 \mu \mathrm{m}$. e, f Quantitative analyses of the lesion depth and mineral

as compared to the group only infected with $S$. mutans/S. sanguinis (Supplementary Fig. S3).

\section{PHR2 is the major contributor to the successful colonization of $C$. albicans in the polymicrobial biofilm under cariogenic condition}

Since response to extreme environmental $\mathrm{pH}$ is critical for the pathogenicity of $C$. albicans, the pH-related genes of $C$. albicans were further investigated in the polymicrobial biofilms. PHR2 gene expression was significantly upregulated among the pH-relative genes of $C$. albicans in the three-species biofilms under cariogenic conditions (Fig. 5a). Deletion of PHR2 loss of the demineralized human enamel blocks by TMR. g Representative photographs of mandibular molars from gingivectomized rats infected with $S$. mutans, S. sanguinis, and/or $C$. albicans for 6 weeks. Black arrows indicated root carious lesions. h Root-caries scores according to Doff's system. $\mathbf{i}, \mathbf{j}$ Levels of $C$. albicans, $S$. mutans, and $S$. sanguinis in in vivo root-caries rat model quantified by qPCR. Data are presented as mean \pm standard deviation $(n=5$ per group; $* * P<$ $0.01 ; * * * P<0.001 ; * * * * P<0.0001 ;$ ns not significant; nd not detectable). S. s S. sanguinis; S. $m$ S. mutans; C. a C. albicans.

significantly suppressed the candidal-streptococcal interactions. Deletion of PHR2 significantly reduced the growth of $C$. albicans in the polymicrobial biofilms as quantified by qPCR and FISH (Fig. 5b-d). In addition, S. mutans/S. sanguinis ratio, as well as the growth of $S$. mutans and $S$. sanguinis in the $S$. mutans/S. sanguinis/phr $2 \Delta / \Delta$ three-species biofilm was comparable to those in the $S$. mutans/S. sanguinis dual species biofilm (Fig. 5c-e and Supplementary Fig. S4a). Deletion of other pH-relative genes such as PHR1, CRZ1, CRZ2, RIM101, RIM8, UME6, TPK1, TPK2, CPH1, and CPH2 also showed a similar trend, but to a lesser extent, regarding the growth of $C$. albicans and streptococcal composition in the three-species biofilms (Fig. 5b, e and Supplementary Fig. S4a). Consistent 


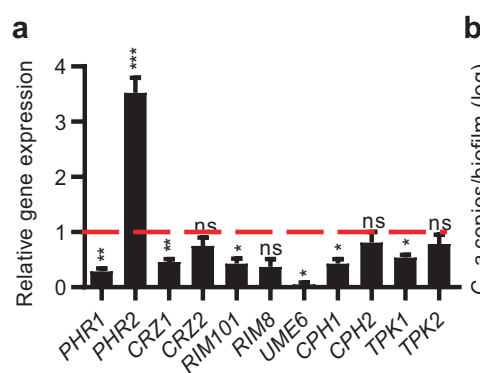

b

d

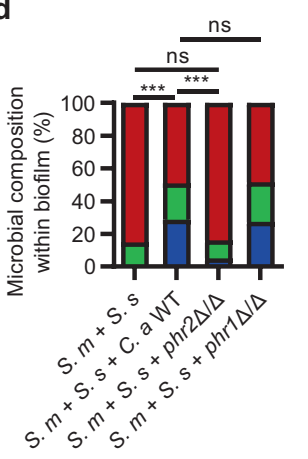

e

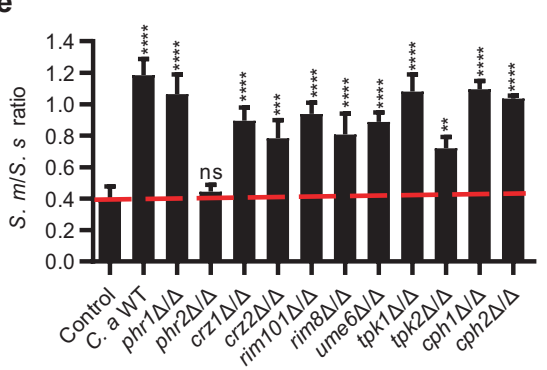

C
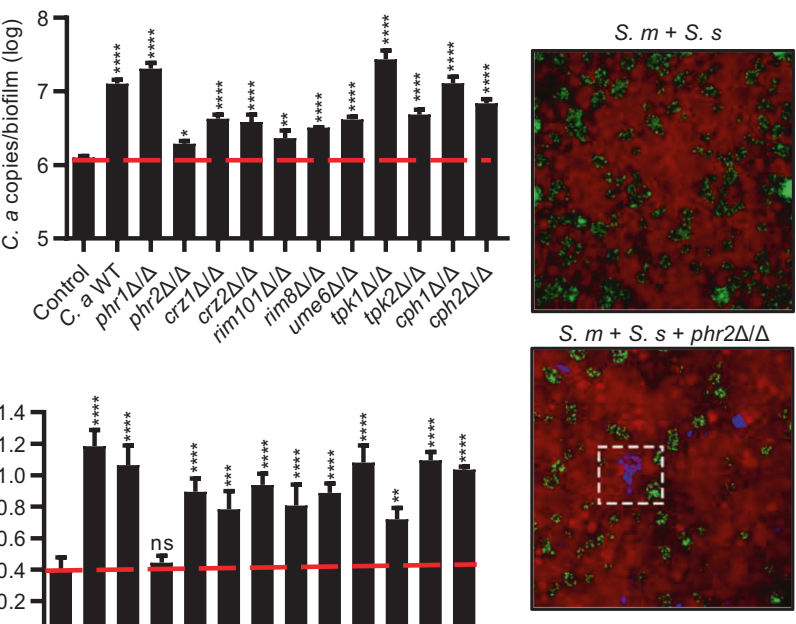

S. $m$

S. $m+S . s$

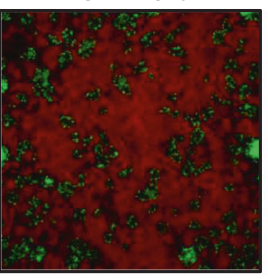

S. $m+$ S. $s+$ C. $a$ WT

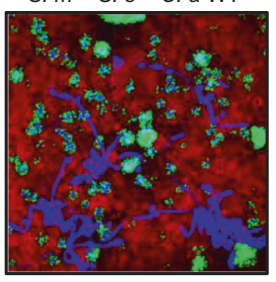

S. $m+S . s+p h r 1 \Delta / \Delta$

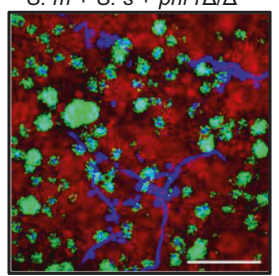

c. a f

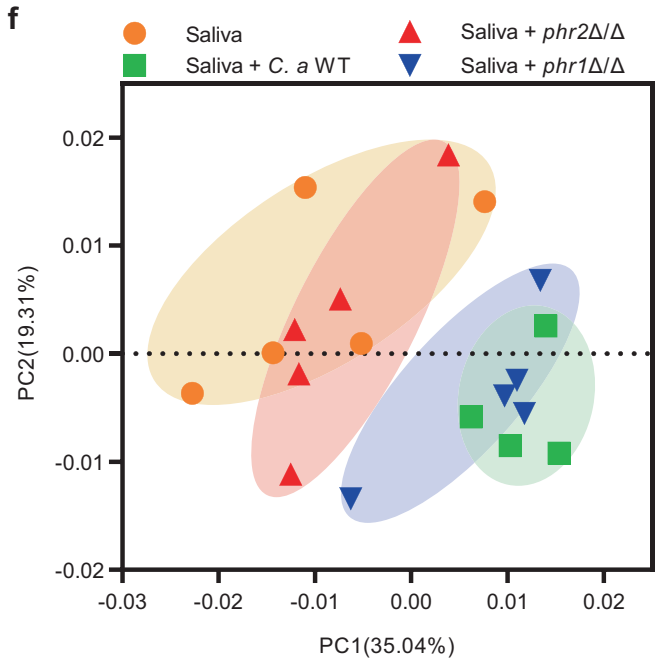

h

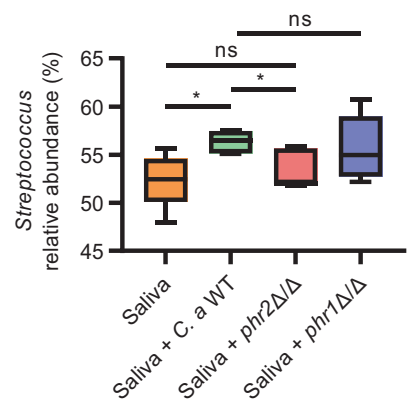

data regarding the growth of each species in the three-species biofilm were observed by CFU counting (Supplementary Fig. S4b-d). g

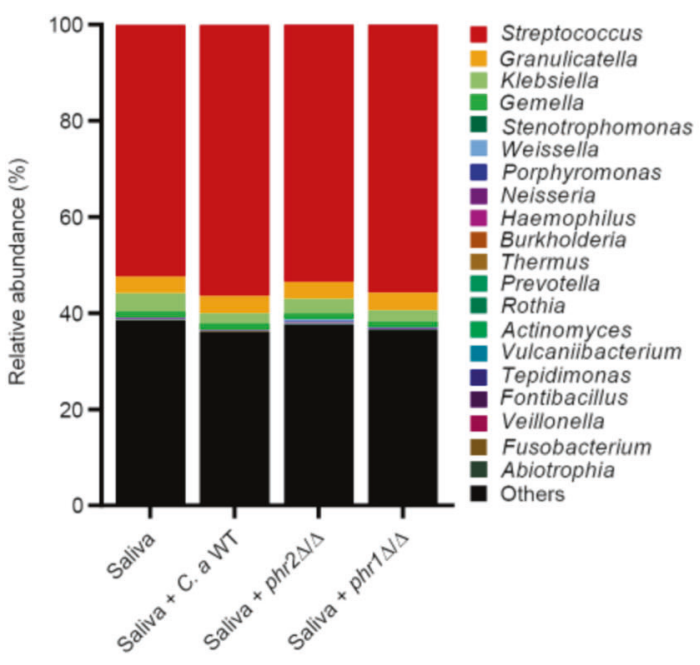

i

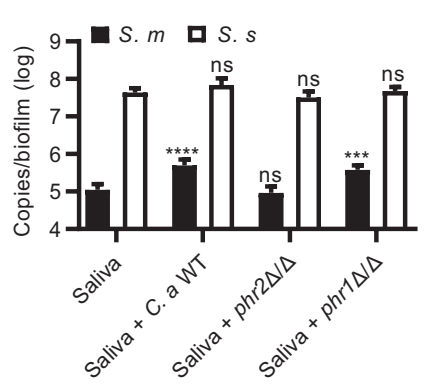

Consistent with data obtained from three-species biofilm, PHR2 gene expression was also significantly upregulated by 13.8 -fold when $C$. albicans was co-cultured with saliva- 
Fig. 5 PHR2 contributes to the success colonization of $C$. albicans in the polymicrobial biofilm under cariogenic condition. a The $\mathrm{pH}-$ relative genes expression of $C$. albicans in the $S$. mutans/S. sanguinis/ C. albicans three-species biofilm. The control group was $C$. albicansonly biofilm. b qPCR quantification of $C$. albicans and its mutants in the three-species biofilm. The control group was $C$. albicans-only biofilm. c Representative images of fluorescence-labelled $S$. mutans (green), S. sanguinis (red), and C. albicans or its PHR1/PHR2 mutants (blue) within three-species biofilm. Scale bar, $60 \mu \mathrm{m}$. d Quantitative analyses of microbial composition in the fluorescence-labelled threespecies biofilm based on IOD. e $S$. mutans/S. sanguinis ratio in the three-species biofilm quantified by qPCR. The control group was $S$. mutans/S. sanguinis two-species biofilm. f PCoA analysis of the polymicrobial oral biofilm in the absence/presence of $C$. albicans or its PHR1/PHR2 deletion mutant. g Relative abundance of the 20 most predominant oral bacteria in the absence/presence of $C$. albicans at genus level. $\mathbf{h}$ The relative abundance of Streptococcus in salivaderived biofilm in the absence/presence of $C$. albicans or its mutants. i Quantification of $S$. mutans and $S$. sanguinis in oral biofilm by qPCR. The control group was saliva-derived biofilm. Data are presented as mean \pm standard deviation (a, b, e: $n=3$ per group; c, d, i: $n=5$ per group; $\mathbf{f}-\mathbf{h}: n=5$ or 4 per group; $* P<0.05$; $* * P<0.01$; *** $P<0.001$; $* * * * P<0.0001$; ns not significant). S. s $S$. sanguinis; S. $m$ S. mutans; C. a C. albicans.

derived biofilm, while PHRl gene expression was unchanged (Supplementary Fig. S4e). Of note, although deletion of $P H R 2$ did not affect the growth of $C$. albicans when grown alone, the growth of $p h r 2 \Delta / \Delta$ was significantly reduced when co-cultured with saliva-derived biofilm as compared to its WT strain (Supplementary Fig. S4f). The impact of $C$. albicans on the microbial ecology of oral biofilm was also PHR2-dependent. Both PCoA (Fig. 5f) and dissimilarity tests (ANOSIM: $R=-0.1360, P=0.914$; Adonis: $R^{2}=0.05396, P=0.968$; Table 1) showed no significant differences in the microbial community structure

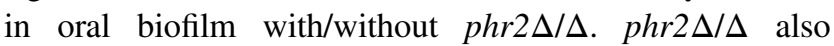
showed little impact on the microbial composition of the oral biofilm, particularly the relative abundance of Streptococcus (Fig. 5g, h and Supplementary Fig. S4g). More importantly, the abundance of $S$. mutans, as well as the $S$. mutans/S. sanguinis ratio, was unchanged when the salivaderived biofilms were grown with $p h r 2 \Delta / \Delta$, as compared with the biofilm free of $C$. albicans (Fig. $5 \mathrm{i}$ and Supplementary Fig. S4h). However, phrl $\Delta / \Delta$ showed similar synergistic interactions with streptococci in the salivaderived biofilm as compared to its WT strain (Fig. 5f-i and Supplementary Fig. S4f-h).

\section{PHR2 deletion deprives C. albicans of the cariogenicity-promoting ability on oral biofilm}

To further investigate whether the cariogenicity-promoting ability of C. albicans on oral biofilm is dependent of PHR2 or not, an in vitro artificial caries model and a root-caries rat model were further used. When the PHR2 was deleted, the cariogenicity-promoting effects of $C$. albicans was abolished, as reflected by the unchanged supernatant $\mathrm{pH}$, ammonia production as well as $l d h$, $\operatorname{arcA}$, or ureC gene expression of saliva-derived biofilms with/without $p h r 2 \Delta / \Delta$ (Fig. 6a, b and Supplementary Fig. S5). Consequently, the saliva-derived biofilm, when co-cultured with $p h r 2 \Delta / \Delta$, induced less demineralization on the tooth hard tissue as compared to the biofilm co-cultured with WT $C$. albicans (Fig. 6c-e). Consistently, the synergistic interactions between $C$. albicans and streptococci on the development of root caries in vivo were also abolished when PHR2 gene was deleted (Fig. 6f-i and Supplementary Fig. S6). Conversely, deletion of PHRI did not alter the cariogenicitypromoting effect of $C$. albicans on oral biofilms (Fig. 6a-i, and Supplementary Figs. S5 and S6).

\section{Discussion}

Dental caries is a result of microbial dysbiosis characterized by the enrichment of acidogenic pathogens and depletion of alkali-generating commensal microbes within the plaque biofilm $[3,50]$. Root caries is a subtype of dental caries that specifically affects the exposed root of tooth, and a significant microbial dysbiosis in the root carious lesions has also been reported [9]. However, factors that drive the microbial alteration which predisposes an individual to root caries are still unclear. We found increased carriage of $C$. albicans in the root-caries lesions, which was positively correlated with increased number of $S$. mutans as well as $S$. mutans/S. sanguinis ratio. Further validation with in vitro polymicrobial biofilm models demonstrated that $C$. albicans colonization caused significant microbial dysbiosis characterized with distinct microbial composition and structure as compared to the biofilm in the absence of $C$. albicans. These findings are in agreement with previous studies showing that $C$. albicans influenced the microbial composition of saliva biofilms [51, 52]. In addition, recent study by Delaney et al. also showed that candida species had a subtle impact on the bacterial microbiome of denture patients and significantly influenced specific genera [53]. Our data showed that C. albicans enriched Streptococcus in the polymicrobial biofilms, particularly increased the ratio of $S$. mutans/S. sanguinis, leading to a more cariogenic biofilm that promoted the development of root caries.

Previous studies showed that $C$. albicans could robustly interact with oral streptococci but with distinct mechanisms. Cell-cell adhesion mediates $C$. albicans/Mitis Group Streptococci interactions [23, 24, 33, 54-58], while EPS plays a critical role in the $C$. albicans/S. mutans interactions $[24,33,54,55,57-59]$ and also contributes to the antifungal resistance of $C$. albicans in the mixed biofilm [60]. Deletion of genes responsible for hyphae formation of $C$. albicans neither reduces microbial carriage nor inhibits 
a

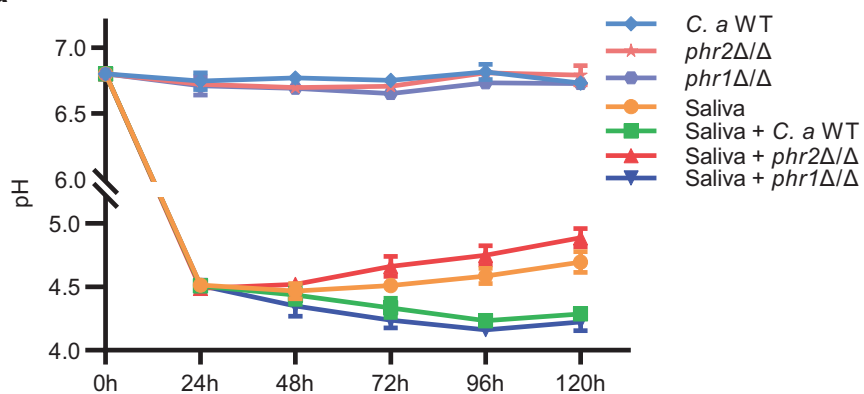

b

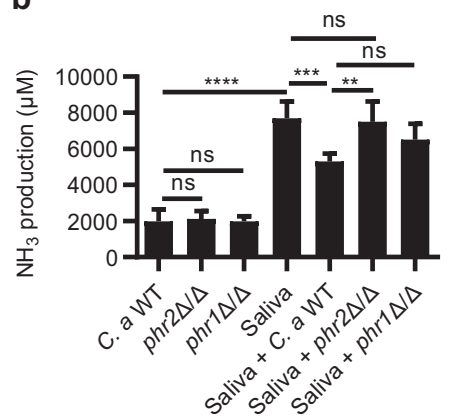

C Saliva

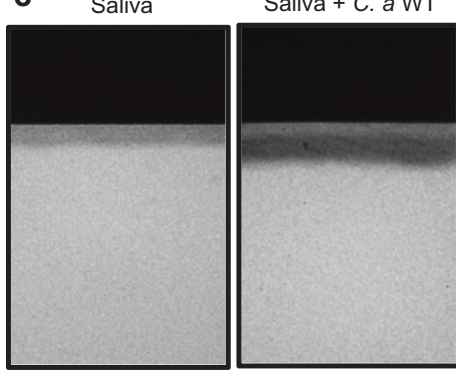

Saliva + C. a WT
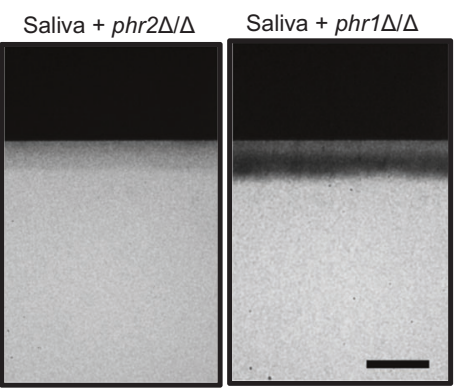

f

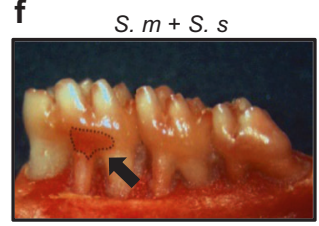

S. $m+$ S. $s+$ phr $2 \Delta / \Delta$
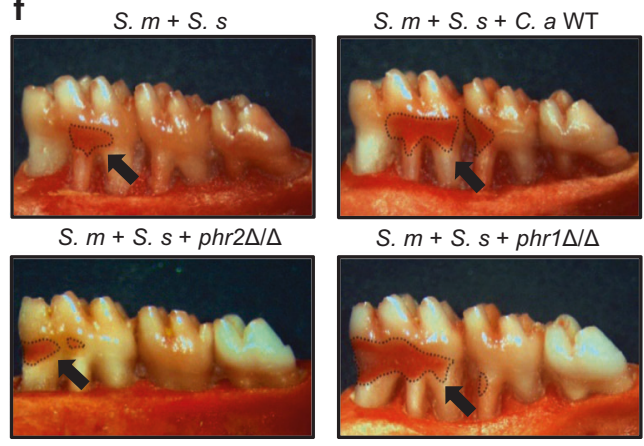

S. $m+S . s+p h r 1 \Delta / \Delta$

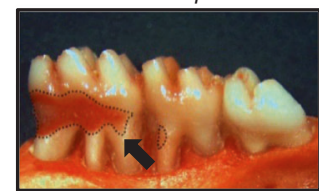

g

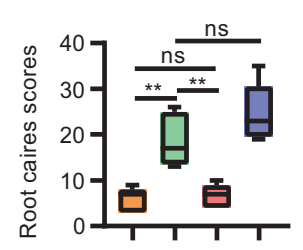

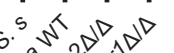

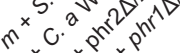

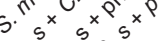

S..$^{x} .5 .5$.

$3 x^{2}+e^{x}$
Fig. 6 PHR2 deletion deprives $C$. albicans of the cariogenicitypromoting ability on oral biofilm. a Dynamic $\mathrm{pH}$ values of the spent media of saliva-derived biofilm in the absence/presence of $C$. albicans or its $P H R 1 / P H R 2$ deletion mutants. b $\mathrm{NH}_{3}$ production by the salivaderived biofilm on the 5 th day after inoculation. $\mathbf{c}$ Representative TMR images of human enamel blocks demineralized by saliva-derived biofilm in the absence/presence of $C$. albicans. Scale bar, $100 \mu \mathrm{m}$. d, e Quantitative analyses of the lesion depth and mineral loss of the demineralized human enamel blocks by TMR. f Representative photographs of mandibular molars from gingivectomized rats infected

cariogenicity of $C$. albicans/S. mutans mixed biofilm [57]. Consistently, the current study also showed that deletion of EFGl and CPHI (key hyphae-associated morphological regulators) [61] did not reduce the number of fungal cells in the polymicrobial biofilm under cariogenic condition as compared to the wild-type $C$. albicans. This is likely due to the fact that the robust production of extracellular $\alpha$-glucans by $S$. mutans via glycosyltransferases (GTFs) under sugarrich condition plays a major role in mediating the coaggregation and biofilm formation of $C$. albicans, which
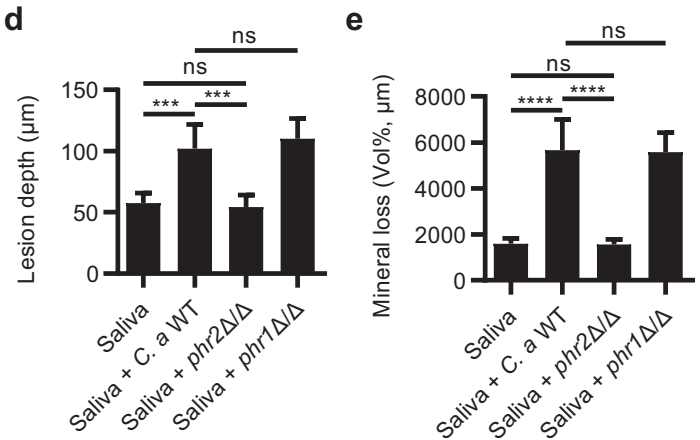

h

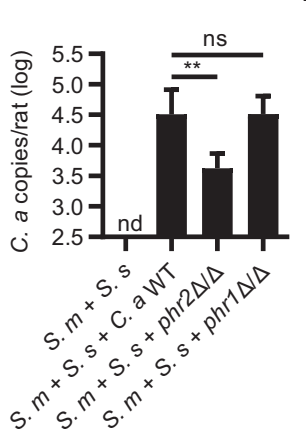

with $S$. mutans, S. sanguinis, and/or $C$. albicans/its mutants for 6 weeks. Black arrows indicated root carious lesions. g Root-caries scores according to Doff's system. h, i Amount of C. albicans/its mutants and $S$. mutans $/ S$. sanguinis in the root-caries rat model quantified by qPCR. The control group of (i) was rats infected with $S$. mutans and $S$. sanguinis. Data are presented as mean \pm standard deviation $(n=5$ per group; $* * P<0.01 ; * * * P<0.001 ; * * * * P<$ 0.0001; ns not significant; nd not detectable). S. s S. sanguinis; S. $m$ S. mutans; C. a C. albicans.

in turn promotes the growth and GTFs expression of $S$. mutans by secreting polysaccharides, quorum sensing molecules as well as metabolic cross-feedings [62-66]. The filamentation-independent synergistic interactions between C. albicans and S. mutans under cariogenic condition ultimately lead to a biofilm with enriched $C$. albicans and acidogenic $S$. mutans, and the latter competes the growth of S. sanguinis and counters the co-adherence of $C$. albicans/S. sanguinis, leading to an unchanged amount of $S$. sanguinis in the mixed microbial consortium. Deletion of EFGl and 
$C P H 1$ can compromise the co-adherence of $C$. albicans/S. sanguinis but still promote the accumulation of $S$. mutans in the polymicrobial biofilm, and this likely explains the reduced amount of $S$. sanguinis observed in the presence of $E F G 1$ and $C P H I$ mutants.

Although the deletion of hyphae-related morphological regulators of $C$. albicans such as $E F G 1$ and $C P H I$ did not affect its co-existence and synergistic interactions with $S$. mutans in the polymicrobial biofilm, the current study found that deletion of $\mathrm{pH}$-relative genes, particularly PHR2 which is associated with acid adaptation of C. albicans, significantly reduced the number of fungal cells in the cariogenic biofilm. PHR1 and PHR2 are functional homologs regulated by extracellular $\mathrm{pH}$ through the Rim 101dependent pathway in opposite ways. PHRl is expressed at $\mathrm{pH}$ values of 5.5 or higher, whereas $P H R 2$ has the reverse expression pattern [67-69]. They are critical factors that determine environment adaptation of $C$. albicans under differed $\mathrm{pH}$ conditions [69-71]. The current study demonstrated that $P H R 2$, rather than PHRI and other pH-relative genes, was significantly upregulated when $C$. albicans was co-cultured with oral biofilms under cariogenic conditions. More importantly, deletion of $P H R 2$ partially restored the microbial ecology of the polymicrobial biofilm, resulting in a biofilm with decreased acidogenicity, compromised demineralizing capability and reduced cariogenicity as recapitulated by the root-caries rat model. It is noteworthy that deletion of PHR2 gene only abolished the cariogenicity-promoting effects of $C$. albicans on the biofilms, but the innate cariogenicity of oral biofilms under cariogenic conditions still existed. These findings suggest that the robust interactions between $C$. albicans with oral streptococci under cariogenic conditions make the traditional hyphae-related virulence genes not the optimal target for the reduction of biofilm cariogenicity at root-caries lesions. However, PHR2 that adapts the co-existence of $C$. albicans with oral streptococci under acidic/cariogenic conditions provides a potential target for the disruption of candidal-streptococcal interactions and their synergistic pathogenicity in the development of root caries.

Taken together, our data demonstrated the critical role of C. albicans infection in development of root caries. $C$. albicans colonization caused significant microbial dysbiosis characterized with an increased abundance of acidogenic/ aciduric $S$. mutans, leading to a more cariogenic biofilm that promoted the development of root caries. PHR2 is the major factor that determines the successful colonization and ecological impact of $C$. albicans in a mixed microbial consortium under cariogenic conditions, and thus may represent a promising target for the control of root caries with candidal involvement. Further study is warranted to investigate the molecular target(s) and signaling pathways via which $C$. albicans binds to and interacts with oral bacteria and consequently elevates the virulence of polymicrobial oral biofilm.

\section{Data availability}

Microbiome sequencing data have been deposited in public database Sequence Read Archive (http://www.ncbi.nlm.nih. gov/Traces/sra) with accession no. PRJNA613946. All data sets generated and/or analyzed in the current study are available from the corresponding author on reasonable request.

Acknowledgements We greatly thank Professors Williama A. Fonzi, Dominique Sanglard, Aaron P. Mitchell, David Kadosh, Joachim F. Ernst, Gerald R. Fink, Haoping Liu and Lo Hsiu-Jung for kindly providing fungal strains. Vivian Chen is a recipient of a 2017-2018 Fulbright Research grant to China. This work was supported by the National Natural Science Foundation of China (81771099 to XX, 81870754 to XZ, 81600858 to BR, 31720103901 to Lixin Zhang), the "111" Project of China (B18022 to Lixin Zhang), a research grant from the Department of Science \& Technology Sichuan Province (2018SZ0121 to XX), and the Fundamental Research Funds for the Central Universities (22221818014S to Lixin Zhang).

Author contributions Conceptualization: QD, BR, L. Zhang, XZ, and XX; methodology: QD, BR, L. Zhang, XZ, and XX; formal analysis: QD, JH, QG, and L. Zheng; investigation: QD, JH, XP, QG; resources: XP, QG, L. Zheng; data curation: JL, HD; writing original draft: QD, BR, XZ, XX; writing, review, and editing: VC, L. Zhang, XZ, XX; visualization: QD, BR, XX; supervision: L. Zhang, XZ; funding acquisition: BR, XZ, XX.

\section{Compliance with ethical standards}

Conflict of interest The authors declare that they have no conflict of interest.

Publisher's note Springer Nature remains neutral with regard to jurisdictional claims in published maps and institutional affiliations.

\section{References}

1. Naghavi M, Abajobir AA, Abbafati C, Abbas KM, Abd-Allah F, Abera SF, et al. Global, regional, and national age-sex specific mortality for 264 causes of death, 1980-2016: a systematic analysis for the Global Burden of Disease Study 2016. Lancet. 2017;390:1151-210.

2. de Olivera Carrilho MR. Root caries: from prevalence to therapy. Karger Medical and Scientific Publishers: Basel, 2017.

3. Pitts NB, Zero DT, Marsh PD, Ekstrand K, Weintraub JA, RamosGomez F, et al. Dental caries. Nat Rev Dis Prim. 2017;3:17030.

4. Kreth J, Merritt J, Shi W, Qi F. Competition and coexistence between Streptococcus mutans and Streptococcus sanguinis in the dental biofilm. J Bacteriol. 2005;187:7193-203.

5. Caufield PW, Dasanayake AP, Li Y, Pan Y, Hsu J, Hardin JM. Natural history of Streptococcus sanguinis in the oral cavity of infants: evidence for a discrete window of infectivity. Infect Immun. 2000;68:4018-23.

6. Mikx FH, van der Hoeven JS, Plasschaert AJ, Konig KG. Establishment and symbiosis of Actinomyces viscosus, 
Streptococcus sanguis and Streptococcus mutans in germ-free Osborne-Mendel rats. Caries Res. 1976;10:123-32.

7. Mitrakul K, Vongsawan K, Sriutai A, Thosathan W. Association between $S$. mutans and $S$. sanguinis in severe early childhood caries and caries-free children a quantitative real-time PCR analysis. J Clin Pediatr Dent. 2016;40:281-9.

8. Loesche W, Rowan J, Straffon L, Loos P. Association of Streptococcus mutans with human dental decay. Infect Immun. 1975;11:1252-60.

9. Chen L, Qin B, Du M, Zhong H, Xu Q, Li Y, et al. Extensive description and comparison of human supra-gingival microbiome in root caries and health. PLoS One. 2015;10:e0117064.

10. Simon-Soro A, Guillen-Navarro M, Mira A. Metatranscriptomics reveals overall active bacterial composition in caries lesions. J Oral Microbiol. 2014;6:25443.

11. Babatzia A, Papaioannou W, Stavropoulou A, Pandis N, KanakaGantenbein C, Papagiannoulis L, et al. Clinical and microbial oral health status in children and adolescents with type 1 diabetes mellitus. Int Dent J. 2020;70:136-44.

12. Zomorodian K, Kavoosi F, Pishdad GR, Mehriar P, Ebrahimi H, Bandegani A, et al. Prevalence of oral Candida colonization in patients with diabetes mellitus. J Mycol Med. 2016;26:103-10.

13. Mun M, Yap T, Alnuaimi AD, Adams GG, McCullough MJ. Oral candidal carriage in asymptomatic patients. Aust Dent J. 2016;61:190-5.

14. Vila T, Sultan AS, Montelongo-Jauregui D, Jabra-Rizk MA. Oral candidiasis: a disease of opportunity. J Fungi. 2020;6:15.

15. Diaz PI, Hong BY, Dupuy AK, Strausbaugh LD. Mining the oral mycobiome: methods, components, and meaning. Virulence. 2017;8:313-23.

16. Shay K, Truhlar MR, Renner RP. Oropharyngeal candidosis in the older patient. J Am Geriatr Soc. 1997;45:863-70.

17. Davis D, Wilson RB, Mitchell AP. RIM101-dependent andindependent pathways govern $\mathrm{pH}$ responses in Candida albicans. Mol Cell Biol. 2000;20:971-8.

18. Calderone RA, Fonzi WA. Virulence factors of Candida albicans. Trends Microbiol. 2001;9:327-35.

19. Gow NA, Brown AJ, Odds FC. Fungal morphogenesis and host invasion. Curr Opin Microbiol. 2002;5:366-71.

20. Shirtliff ME, Peters BM, Jabra-Rizk MA. Cross-kingdom interactions: Candida albicans and bacteria. FEMS Microbiol Lett. 2009;299:1-8.

21. Bamford CV, d'Mello A, Nobbs AH, Dutton LC, Vickerman MM, Jenkinson HF. Streptococcus gordonii modulates Candida albicans biofilm formation through intergeneric communication. Infect Immun. 2009;77:3696-704.

22. Morales DK, Hogan DA. Candida albicans interactions with bacteria in the context of human health and disease. PLoS Pathog. 2010;6:e1000886.

23. Diaz PI, Xie Z, Sobue T, Thompson A, Biyikoglu B, Ricker A, et al. Synergistic interaction between Candida albicans and commensal oral streptococci in a novel in vitro mucosal model. Infect Immun. 2012;80:620-32.

24. Koo H, Andes DR, Krysan DJ. Candida-streptococcal interactions in biofilm-associated oral diseases. PLoS Pathog. 2018;14: e1007342.

25. Xu H, Sobue T, Thompson A, Xie Z, Poon K, Ricker A, et al. Streptococcal co-infection augments Candida pathogenicity by amplifying the mucosal inflammatory response. Cell Microbiol. 2014;16:214-31.

26. Xu H, Sobue T, Bertolini M, Thompson A, Dongari-Bagtzoglou A. Streptococcus oralis and Candida albicans synergistically activate mu-calpain to degrade E-cadherin from oral epithelial junctions. J Infect Dis. 2016;214:925-34.

27. Xu H, Sobue T, Bertolini M, Thompson A, Vickerman M, Nobile CJ, et al. S. oralis activates the Efg1 filamentation pathway in C. albicans to promote cross-kingdom interactions and mucosal biofilms. Virulence. 2017;8:1602-17.

28. Lozano Moraga CP, Rodriguez Martinez GA, Lefimil Puente CA, Morales Bozo IC, Urzua, Orellana BR. Prevalence of Candida albicans and carriage of Candida non-albicans in the saliva of preschool children, according to their caries status. Acta Odontol Scand. 2017;75:30-5.

29. Wu N, Lin J, Wu L, Zhao J. Distribution of Candida albicans in the oral cavity of children aged 3-5 years of Uygur and Han nationality and their genotype in caries-active groups. Genet Mol Res. 2015;14:748-57.

30. Xiao J, Moon Y, Li L, Rustchenko E, Wakabayashi H, Zhao X, et al. Candida albicans carriage in children with severe early childhood caries (S-ECC) and maternal relatedness. PLoS One. 2016;11:e0164242.

31. Xiao J, Grier A, Faustoferri RC, Alzoubi S, Gill AL, Feng C, et al. Association between oral candida and bacteriome in children with severe ECC. J Dent Res. 2018;97:1468-76.

32. Xiao J, Huang X, Alkhers N, Alzamil H, Alzoubi S, Wu TT, et al. Candida albicans and early childhood caries: a systematic review and meta-analysis. Caries Res. 2018;52:102-12.

33. Falsetta ML, Klein MI, Colonne PM, Scott-Anne K, Gregoire S, Pai $\mathrm{CH}$, et al. Symbiotic relationship between Streptococcus mutans and Candida albicans synergizes virulence of plaque biofilms in vivo. Infect Immun. 2014;82:1968-81.

34. Beighton D, Lynch E. Relationships between yeasts and primary root-caries lesions. Gerodontology. 1993;10:105-8.

35. Shen S, Samaranayake LP, Yip HK. Coaggregation profiles of the microflora from root surface caries lesions. Arch Oral Biol. 2005;50:23-32.

36. Zaremba ML, Stokowska W, Klimiuk A, Daniluk T, Rozkiewicz D, Cylwik-Rokicka D, et al. Microorganisms in root carious lesions in adults. Adv Med Sci. 2006;51 Suppl 1:237-40.

37. Dige I, Nyvad B. Candida species in intact in vivo biofilm from carious lesions. Arch Oral Biol. 2019;101:142-6.

38. Ev LD, Dame-Teixeira N, Do T, Maltz M, Parolo CCF. The role of Candida albicans in root caries biofilms: an RNA-seq analysis. J Appl Oral Sci. 2020;28:e20190578.

39. Edlund A, Yang Y, Hall AP, Guo L, Lux R, He X, et al. An in vitro biofilm model system maintaining a highly reproducible species and metabolic diversity approaching that of the human oral microbiome. Microbiome. 2013;1:25.

40. Tian Y, He X, Torralba M, Yooseph S, Nelson KE, Lux R, et al. Using DGGE profiling to develop a novel culture medium suitable for oral microbial communities. Mol Oral Microbiol. 2010;25:357-67.

41. Zhang K, Ren B, Zhou X, Xu HH, Chen Y, Han Q, et al. Effect of antimicrobial denture base resin on multi-species biofilm formation. Int J Mol Sci. 2016;17:e1033.

42. Shannon P, Markiel A, Ozier O, Baliga NS, Wang JT, Ramage D, et al. Cytoscape: a software environment for integrated models of biomolecular interaction networks. Genome Res. 2003;13:2498-504.

43. Langille MG, Zaneveld J, Caporaso JG, McDonald D, Knights D, Reyes JA, et al. Predictive functional profiling of microbial communities using $16 \mathrm{~S}$ rRNA marker gene sequences. Nat Biotechnol. 2013;31:814-21.

44. Moyes DL, Wilson D, Richardson JP, Mogavero S, Tang SX, Wernecke J, et al. Candidalysin is a fungal peptide toxin critical for mucosal infection. Nature. 2016;532:64-8.

45. Klug B, Rodler C, Koller M, Wimmer G, Kessler HH, Grube M, et al. Oral biofilm analysis of palatal expanders by fluorescence insitu hybridization and confocal laser scanning microscopy. J Vis Exp. 2011;56:e2967-e.

46. Xiao J, Klein MI, Falsetta ML, Lu B, Delahunty CM, Yates JR III, et al. The exopolysaccharide matrix modulates the interaction 
between 3D architecture and virulence of a mixed-species oral biofilm. PLoS Pathog. 2012;8:e1002623.

47. Du Q, Fu M, Zhou Y, Cao Y, Guo T, Zhou Z, et al. Sucrose promotes caries progression by disrupting the microecological balance in oral biofilms: an in vitro study. Sci Rep. 2020;10:2961.

48. Firestone AR, Graves CN, Feagin FF. The effects of different levels of dietary sucrose on root caries subsequent to gingivectomy in conventional rats infected with Actinomyces viscosus M-100. J Dent Res. 1988;67:1342-5.

49. Doff RS, Rosen S, App G. Root surface caries in the molar teeth of Rice rats. I. A method for quantitative scoring. J Dent Res. 1977;56:1013-6.

50. Xu X, Chen F, Huang Z, Ma L, Chen L, Pan Y, et al. Meeting report: a close look at oral biofilms and microbiomes. Int $\mathrm{J}$ Oral Sci. 2018;10:28.

51. Lambooij JM, Hoogenkamp MA, Brandt BW, Janus MM, Krom BP. Fungal mitochondrial oxygen consumption induces the growth of strict anaerobic bacteria. Fungal Genet Biol. 2017;109:1-6.

52. Janus MM, Crielaard W, Volgenant CM, van der Veen $\mathrm{MH}$, Brandt BW, Krom BP. Candida albicans alters the bacterial microbiome of early in vitro oral biofilms. J Oral Microbiol. 2017;9:1270613.

53. Delaney C, O'Donnell LE, Kean R, Sherry L, Brown JL, Calvert $\mathrm{G}$, et al. Interkingdom interactions on the denture surface: implications for oral hygiene. Biofilm. 2019;1:100002.

54. Hwang G, Marsh G, Gao L, Waugh R, Koo H. Binding force dynamics of Streptococcus mutans-glucosyltransferase B to Candida albicans. J Dent Res. 2015;94:1310-7.

55. Gregoire S, Xiao J, Silva BB, Gonzalez I, Agidi PS, Klein MI, et al. Role of glucosyltransferase B in interactions of Candida albicans with Streptococcus mutans and with an experimental pellicle on hydroxyapatite surfaces. Appl Environ Microbiol. 2011;77:6357-67.

56. Bowen WH, Koo H. Biology of Streptococcus mutans-derived glucosyltransferases: role in extracellular matrix formation of cariogenic biofilms. Caries Res. 2011;45:69-86.

57. Hwang G, Liu Y, Kim D, Li Y, Krysan DJ, Koo H. Candida albicans mannans mediate Streptococcus mutans exoenzyme GtfB binding to modulate cross-kingdom biofilm development in vivo. PLoS Pathog. 2017;13:e1006407.

58. Kim D, Koo H. Spatial design of polymicrobial oral biofilm in its native disease state. J Dent Res. 2020;99:597-603.

59. Bowen WH, Burne RA, Wu H, Koo H. Oral biofilms: pathogens, matrix, and polymicrobial interactions in microenvironments. Trends Microbiol. 2018;26:229-42.
60. Kim D, Liu Y, Benhamou RI, Sanchez H, Simon-Soro A, Li Y, et al. Bacterial-derived exopolysaccharides enhance antifungal drug tolerance in a cross-kingdom oral biofilm. ISME J. 2018;12:1427-42.

61. Nobile CJ, Fox EP, Nett JE, Sorrells TR, Mitrovich QM, Hernday $\mathrm{AD}$, et al. A recently evolved transcriptional network controls biofilm development in Candida albicans. Cell. 2012;148:126-38.

62. Kim D, Sengupta A, Niepa TH, Lee BH, Weljie A, Freitas-Blanco VS, et al. Candida albicans stimulates Streptococcus mutans microcolony development via cross-kingdom biofilm-derived metabolites. Sci Rep. 2017;7:41332.

63. Sztajer H, Szafranski SP, Tomasch J, Reck M, Nimtz M, Rohde $\mathrm{M}$, et al. Cross-feeding and interkingdom communication in dualspecies biofilms of Streptococcus mutans and Candida albicans. ISME J. 2014;8:2256-71.

64. Rossi DCP, Gleason JE, Sanchez H, Schatzman SS, Culbertson EM, Johnson CJ, et al. Candida albicans FRE8 encodes a member of the NADPH oxidase family that produces a burst of ROS during fungal morphogenesis. PLoS Pathog. 2017;13:e1006763.

65. Ellepola K, Truong T, Liu Y, Lin Q, Lim TK, Lee YM, et al. Multi-omics analyses reveal synergistic carbohydrate metabolism in Streptococcus mutans-Candida albicans mixed-species biofilms. Infect Immun. 2019;87:e00339-19.

66. Khoury ZH, Vila T, Puthran TR, Sultan AS, Montelongo-Jauregui D, Melo MAS, et al. The role of Candida albicans secreted polysaccharides in augmenting Streptococcus mutans adherence and mixed biofilm formation: in vitro and in vivo studies. Front Microbiol. 2020;11:307.

67. Baek YU, Martin SJ, Davis DA. Evidence for novel pHdependent regulation of Candida albicans Rim101, a direct transcriptional repressor of the cell wall beta-glycosidase Phr2. Eukaryot Cell. 2006;5:1550-9.

68. Muhlschlegel FA, Fonzi WA. PHR2 of Candida albicans encodes a functional homolog of the pH-regulated gene PHR1 with an inverted pattern of $\mathrm{pH}$-dependent expression. Mol Cell Biol. 1997;17:5960-7.

69. Saporito-Irwin SM, Birse CE, Sypherd PS, Fonzi WA. PHR1, a $\mathrm{pH}$-regulated gene of Candida albicans, is required for morphogenesis. Mol Cell Biol. 1995;15:601-13.

70. De Bernardis F, Muhlschlegel FA, Cassone A, Fonzi WA. The pH of the host niche controls gene expression in and virulence of Candida albicans. Infect Immun. 1998;66:3317-25.

71. Calderon J, Zavrel M, Ragni E, Fonzi WA, Rupp S, Popolo L. PHR1, a pH-regulated gene of Candida albicans encoding a glucan-remodelling enzyme, is required for adhesion and invasion. Microbiology. 2010;156:2484-94. 